Moroccan J. of Pure and Appl. Anal. (MJPAA)

Volume 7(1), 2021, Pages 66-79

ISSN: Online 2351-8227 - Print 2605-6364

DOI: $10.2478 / \mathrm{mjpaa}-2021-0007$

\title{
Optimal control strategy of COVID-19 spread in Morocco using SEIRD model
}

\author{
H. FERJOUCHIA ${ }^{1}$, A. KOUIDERE ${ }^{2}$, O. ZAKARY ${ }^{3}$, AND M. RACHIK ${ }^{4}$
}

\begin{abstract}
Aвstract. This paper aims to predict the development of the COVID-19 pandemic in Morocco from a mathematical approach. Based on the reliability of the data and the nature of confirmed cases, the SEIRD model is employed to provide a theoretical framework to forecast COVID-19 ongoing epidemic. Findings suggest that the structure and parameters of the proposed model give insights into the dynamics of the virus. Hence, this study contributes to the conceptual areas of knowledge on COVID-19 in proposing an optimal control plan to help decrease the number of confirmed cases by applying preventive measures such as social distancing, wearing facial masks. Matlab/ Simulink TM simulations are used to illustrate the findings.
\end{abstract}

Mathematics Subject Classification (2020). 93A30, $49 \mathrm{~J} 15$.

Key words and phrases. COVID-19, Dynamic model, Epidemiological models, Optimal Control and Pontryagin's Maximum Principle.

\section{Introduction}

Coronavirus disease (COVID-19) is a potentially severe respiratory disease that can spread from a person to another. COVID-19 is a novel coronavirus that was first identified in Wuhan China through retrospective investigations by Chinese authorities. Because the virus is highly

Received : 07 July 2020 - Accepted: 20 October 2020.

(C) The Author(s) 2020. This article is published with open access by Sidi Mohamed Ben Abdallah University. 1,2,3,4 Laboratory Analysis, Modeling and Simulation LAMS. Department of Mathematics and Computer Science, Faculty of Sciences Ben MSik, Hassan II University of Casablanca, Morocco.

e-mail ${ }^{1}$ : ferjouchiahanane@gmail.com (Corresponding Author).

e-mail2: kouidere89@gmail.com

e-mail ${ }^{3}$ : zakaryma@gmail.com

e-mail ${ }^{4}:$ m_rachik@yahoo.fr. 
contagious, it spread rapidly across the world at a rapid rate resulting in an ongoing pandemic to become the first global outbreak caused by a coronavirus $[16,20]$. The virus spreads primarily via small respiratory droplets expelled when an infected person by coughing, or sneezing. Transmission may also occur through can touching contaminated surface or objects followed by touching their eyes, noses or mouths before washing their hands. However, indirect transmission of COVID-19 is considered less robust than the evidence of direct contact transmission [5].

In December 2019, the first case of a novel coronavirus was identified in Wuhan, China [11]. The disease subsequently spread rapidly worldwide. The World Health Organization (WHO) termed the outbreak 2019 novel coronavirus (COVID-19) ON February 2020. China reported about 10 thousand confirmed cases $(9,720)$ from December 2019 to 31 January 2020 [17]. It is worth mentioning that asymptomatically infected individuals can spread the virus to others $[18,23]$. This suggests that detecting more cases of COVID-19 and effectively identify asymptomatic people would prove to be a challenge for medical authorities. In the absence of a vaccine, or effective therapeutics against COVID-19, one of our only remaining strategies for controlling COVID-19 is to resort to non-pharmaceutical interventions to decrease the spread of COVID-19 in the community.

Morocco is among the countries affected by COVID-19. As of June 10, 2020, Morocco reported a total of 8508 confirmed cases and 211 deaths [4]. The first COVID-19 case in Morocco was detected on March 02, 2020 in Casablanca city and It involved a Moroccan citizen importing the virus from Italy[4]. As the virus spread widened in Morocco, in mid-March the Government closed schools and classes continued through online interactive learning as an alternative [21]. Morocco declared a state of emergency nationwide that required significant restrictions on the movement of people and banning social gatherings of more than people [29]. Mosques and non-essential businesses were closed. However, essential or urgent public support services such as pharmacies and stores selling necessary goods to citizens remained opened.

In epidemiology, various mathematical models have been used to describe epidemic processes and understand the temporal dynamics of infectious diseases. Many contemporary mathematical models used to describe the transmission of infectious diseases are based on the basic compartmental models by Kermack and McKendrick (1927) and Kermack and McKendrick (1932). Compartmental models which are based on a system a system of ordinary differential equations

describing the change in the population size of each compartment. These models have been widely investigated and have benefited also from major advances in computational power over the past several decades. The SEIRD epidemic used in this paper is one of the simplest forms of these compartmental models.

Comprehensive textbook in field of epidemiology and Imperial college COVID-19 Response

Team (2020) serve as examples of how these mathematical models have been applied by epidemiologists in the effort to understand the current health crisis.

By using cumulative data of confirmed COVID-19 cases in Morocco between 20 April 2020 
and 10 June 2020 (See Table 1), we proposed in this study a modified mathematical model that takes into account the specificity of the Moroccan pandemic situation and attempts to assess the efficiency of the control measures imposed by the Moroccan government on 20 March to limit the spread of the virus in the country. The implemented model computes the basic reproduction number and determines the impact of the control measures on the peak time, the duration of the epidemic, peak size and the size of the epidemic. This paper can help provide a more complete understanding of the disease progression and adjust disease intervention strategies in Morocco.

\begin{tabular}{|c|c|c|c|c|}
\hline Date (day/month) & Daily Cases & Cumulative cases & Daily Deaths & Cumulative Deaths \\
\hline 20 April & 3046 & 191 & 2 & 143 \\
\hline 21 April & 3209 & 163 & 2 & 143 \\
\hline 22 April & 3446 & 237 & 4 & 149 \\
\hline 23 April & 3568 & 122 & 6 & 155 \\
\hline 24 April & 3758 & 190 & 3 & 158 \\
\hline 25 April & 3897 & 139 & 1 & 159 \\
\hline 26 April & 4065 & 168 & 2 & 161 \\
\hline 27 April & 4120 & 55 & 1 & 162 \\
\hline 28 April & 4252 & 132 & 3 & 165 \\
\hline 29 April & 4321 & 61 & 3 & 168 \\
\hline 30 April & 4423 & 120 & 2 & 170 \\
\hline 1 May & 4569 & 146 & 1 & 171 \\
\hline 2 May & 4729 & 160 & 2 & 173 \\
\hline 3 May & 4903 & 174 & 1 & 174 \\
\hline 4 May & 5053 & 150 & 5 & 179 \\
\hline 5 May & 5219 & 166 & 2 & 181 \\
\hline 6 May & 5408 & 189 & 2 & 185 \\
\hline 7 May & 5548 & 140 & 0 & 183 \\
\hline 8 May & 5711 & 163 & 3 & 186 \\
\hline 9 May & 5910 & 199 & 0 & 186 \\
\hline 10 May & 6063 & 153 & 2 & 188 \\
\hline 11 May & 6281 & 218 & 0 & 188 \\
\hline 12 May & 6418 & 137 & 0 & 188 \\
\hline 13 May & 6512 & 94 & 0 & 188 \\
\hline 14 May & 6607 & 95 & 2 & 190 \\
\hline 15 May & 6652 & 45 & 0 & 190 \\
\hline 16 May & 6741 & 89 & 2 & 192 \\
\hline 17 May & 6870 & 129 & 0 & 192 \\
\hline 18 May & 6952 & 82 & 0 & 192 \\
\hline 19 May & 7023 & 71 & 1 & 193 \\
\hline 20 May & 7133 & 110 & 1 & 194 \\
\hline 21 May & 7211 & 78 & 2 & 196 \\
\hline
\end{tabular}




\begin{tabular}{ccccc}
\hline Date (day/month) & Daily Cases & Cumulative cases & Daily Deaths & Cumulative Deaths \\
\hline 22 May & 7332 & 121 & 1 & 197 \\
23 May & 7406 & 74 & 1 & 198 \\
24 May & 7433 & 27 & 1 & 199 \\
25 May & 7532 & 99 & 1 & 200 \\
26 May & 7577 & 45 & 2 & 202 \\
27 May & 7601 & 24 & 0 & 202 \\
28 May & 7643 & 42 & 0 & 202 \\
29 May & 7714 & 71 & 0 & 204 \\
30 May & 7780 & 66 & 2 & 205 \\
31 May & 7807 & 27 & 2 & 205 \\
1 June & 7833 & 26 & 0 & 206 \\
2 June & 7866 & 33 & 1 & 206 \\
3 June & 7922 & 56 & 0 & 208 \\
4 June & 8003 & 81 & 2 & 208 \\
5 June & 8071 & 68 & 0 & 208 \\
6 June & 8151 & 80 & 0 & 208 \\
7 June & 8224 & 73 & 0 & 210 \\
8 June & 8302 & 78 & 0 & 211 \\
9 June & 8437 & 135 & 2 & 1 \\
10 June & 8508 & 71 & 19 &. \\
\hline \hline
\end{tabular}

TABLE 1. Parameter values of the COVID-19 taken from Reference [4].

This study uses the accumulated data of confirmed COVID-19 cases in Morocco between 20 April 2020 and 10 June 2020.

Factors such as birth rate and natural diseases are excluded are not considered so the total population remains constant.

The primary purpose of this study is to present control strategies to determine the transmission dynamics of COVID-19 in Morocco. The remainder of the paper is organized as follows. Section 2 presents an adaptation of SEIRD model used to calculate the spread of COVID-19 in Morocco. Section 3 formulates an optimal control model for COVID-19 illustrates numerical simulations. Section 4 concludes.

\section{Normalized SEIRD COVID-19 Model}

The SEIRD epidemic model for COVID-19 transmission suggested in References [24] [25]. is expressed by the following system of ordinary differential equations:

$$
\left\{\begin{array}{l}
S^{\prime}(t)=-\beta S(t) I(t) \\
E^{\prime}(t)=\beta S(t) I(t)-\eta E(t) \\
I^{\prime}(t)=\eta E(t)-\delta I(t) \\
R^{\prime}(t)=\delta(1-\gamma) I(t) \\
D^{\prime}(t)=\delta \gamma I(t)
\end{array}\right.
$$



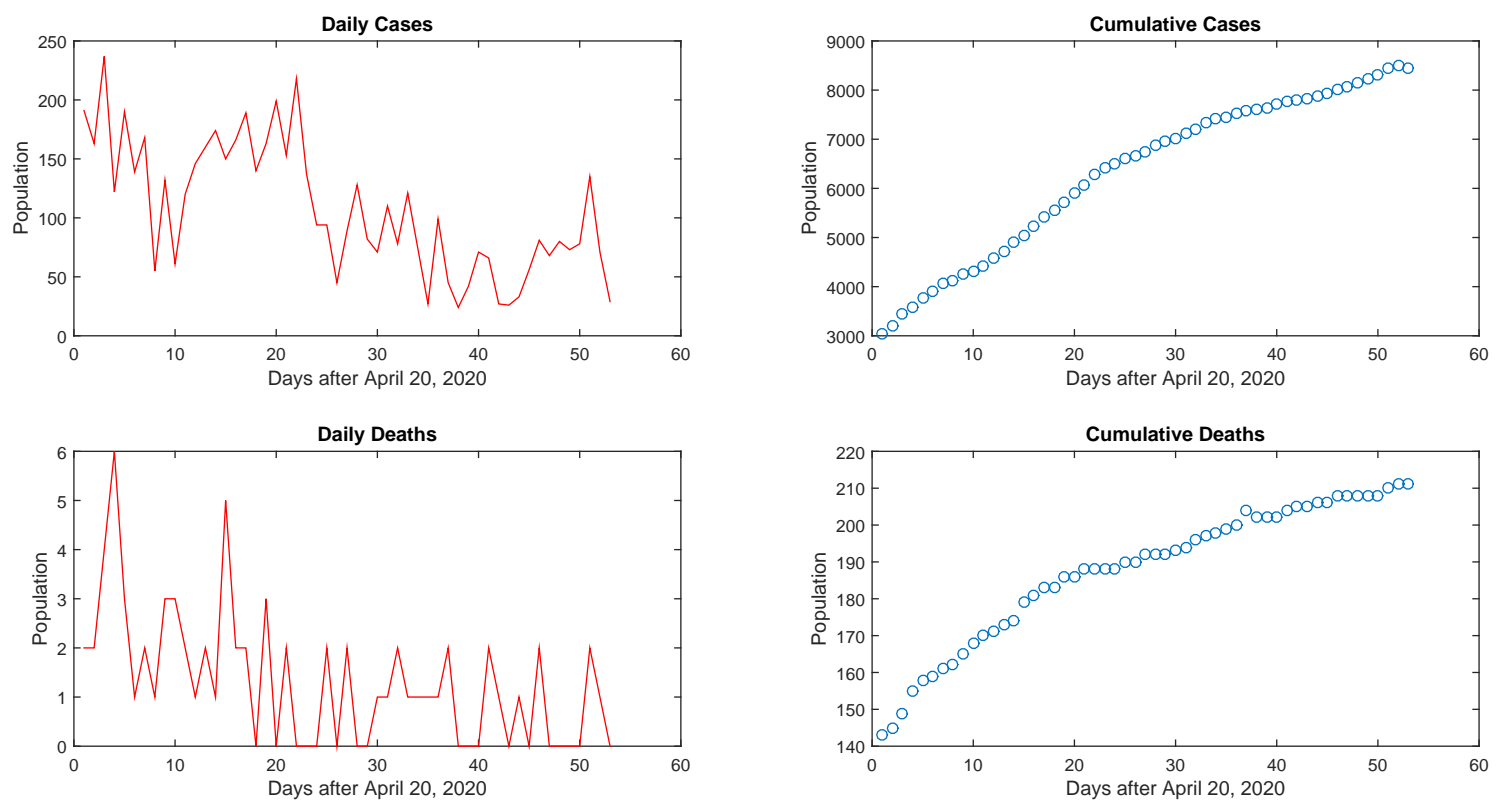

Figure 1. Confirmed cases and death cases from 20 April 2020 to 10 June 2020

The model in Equation $\left(S_{1}\right)$ subdivides human population into five mutually exclusive compartments:

- Susceptible(S) Those who have not been infected by the virus yet but can become infected.

- Exposed(E) Those who are infected by the virus and still in the incubation phase. Therefore, they cannot transmit it to others yet.

- Infectious(I) Those infected by the disease and can transmit it.

- Recovered(R) Those who were infected by the disease by recovered.

- Dead(D) Those who died because of the virus.

Each population represents a proportion of the total population. The total population at time $t$, denoted by $N(t)$, is given by $N(t)=S(t)+E(t)+I(t)+R(t)+D(t)$.

Figure. 2 provides a schematic representation of model Interactions between the compartments of the epidemiological model $\left(S_{1}\right)$. The arrows represent transition between compartments. The evolution of the SEIRD model depends on several parameters. All the parameters are nonnegative constants, and they are described in table 2.

\begin{tabular}{cl}
\hline Parameter & Description \\
\hline$\beta$ & the rate at which infectious people interact with others \\
$\eta$ & the incubation rate \\
$\delta$ & the estimated duration of illness \\
$\gamma$ & the case fatality ratio \\
$r$ & the proportion of all COVID-19 cases that is observed \\
\hline
\end{tabular}




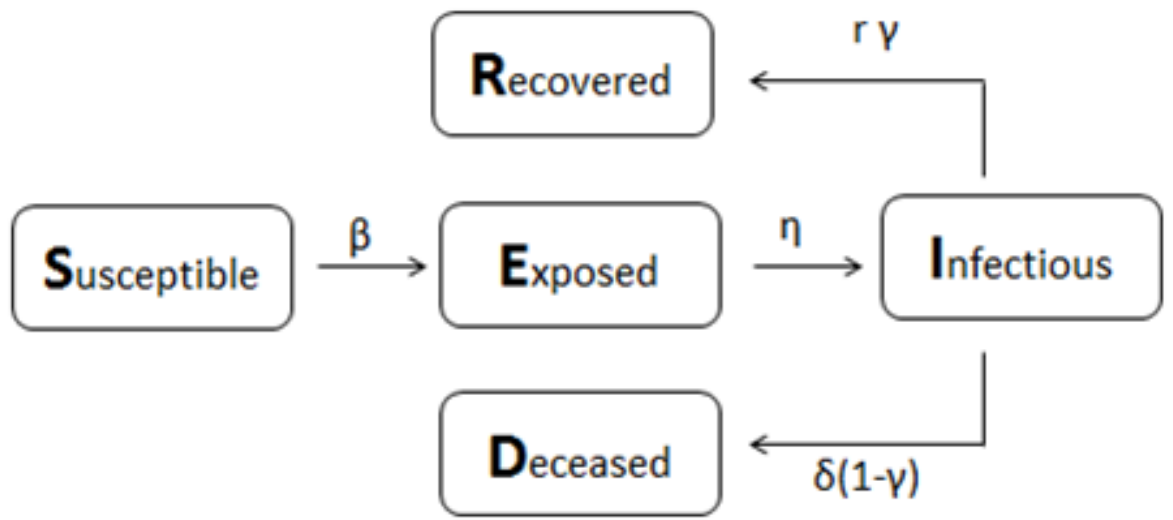

Figure 2. A compartmental diagram of COVID-19 transmission model

TABLE 2. Parameter description of the COVID-19 model in Equation $\left(S_{1}\right)$

This study is based on the data of confirmed COVID-19 cases in Morocco. It consists of the daily reported number of new cases and accumulated cases of COVID-19 from 20 April to 10 June 2020 (see Figure 1 and Table 1). The parameter $\beta$ reflects the rate at which infectious people interact with others. It is often written as $\beta=\mathcal{R}_{0} \delta$, where $\mathcal{R}_{0}$, called the basic reproduction number, measures the transmission of the disease with no mitigation efforts. The classical SEIRD model demonstrates that asymptotic behaviour depends in the basic reproduction of $\mathcal{R}_{0}$ the expected number of secondary cases produced an infective person in a completely suspectable population). It is described as a threshold value that indicates whether the initial outbreak occurs or not. That is, if $\mathcal{R}_{0}<1$, then the number of the infected population decreases, and the outbreak eventually peter out on its own. whereas if $\mathcal{R}_{0}>1$, then the number of the infected population to increase exponentially, and an epidemic could ensue.

In Morocco, the optimal estimates of $\mathcal{R}_{0}$ of COVID-19 ranges between 1.22 and 2.55 [1]. In the context of this paper, the transmissibility if COVID-19 and median are calculated around $\mathcal{R}_{0}=1.37$.

The study is based on official reports of the total number of cases and the numbers of infected individuals and the number of deceased individuals (From 20 April to 10 June). The complete data sets are listed in Table 1.

Numerical integration and simulation, the model formulation and the complete implementation are carried in the MATLAB code.

Listing 1. The ODE function.

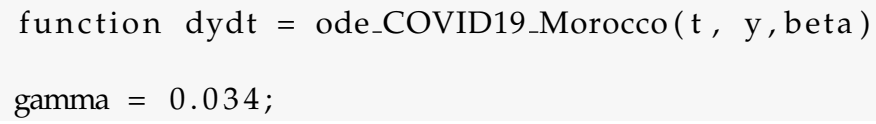




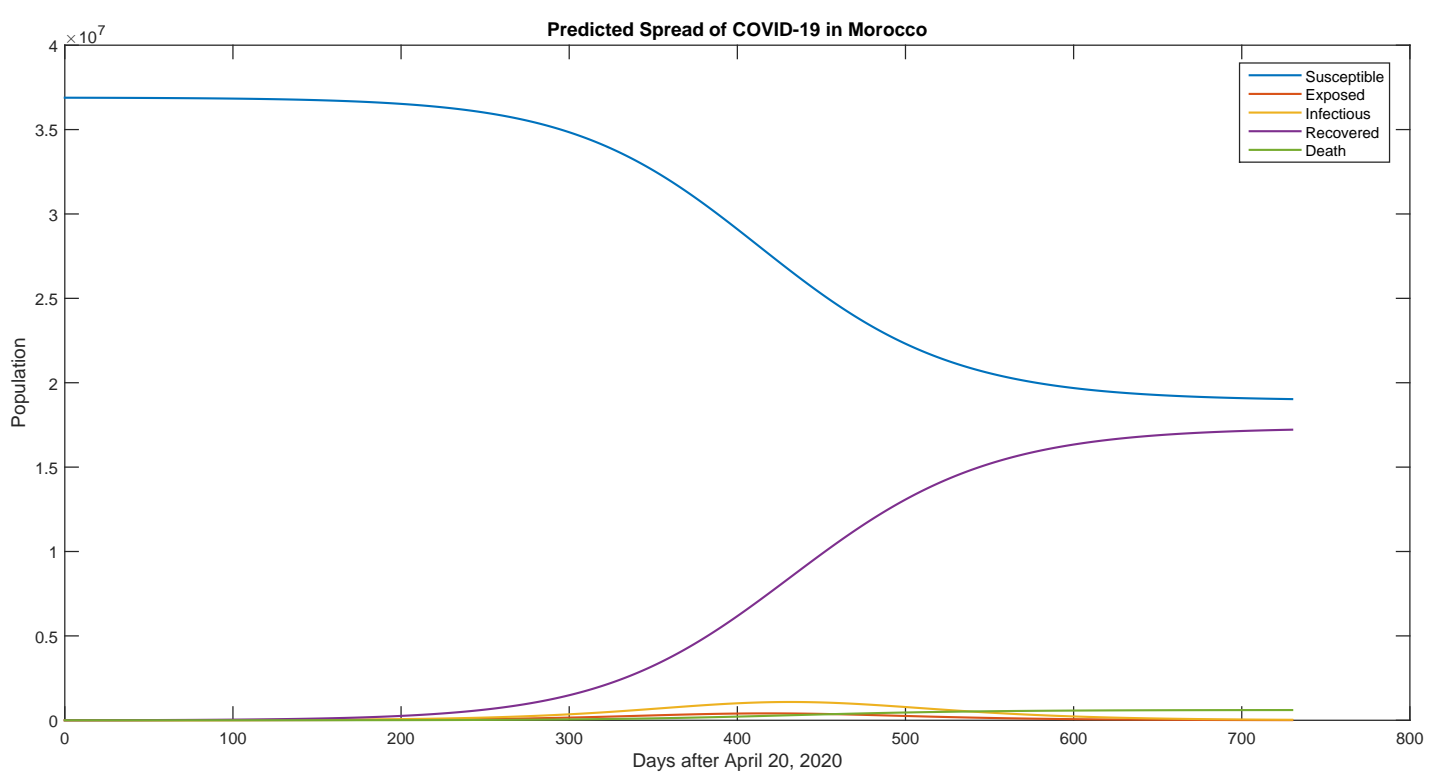

FiguRE 3. Predicted Spread of COVID-19 in Morocco for 2 years

$$
\left\{\begin{array}{l}
S^{\prime}(t)=-\beta(1-u(t)) S(t) I(t) \\
E^{\prime}(t)=\beta(1-u(t)) S(t) I(t)-\eta E(t) \\
I^{\prime}(t)=\eta E(t)-\delta I(t) \\
R^{\prime}(t)=\delta(1-\gamma) I(t) \\
D^{\prime}(t)=\delta \gamma I(t)
\end{array}\right.
$$

The control $u(.) \mathrm{u}($.$) is bounded between 0$ and $u_{\max }$, with $u_{\max }<1$. When the control vanishes, no extra preventive measure for COVID-19 transmission is used by susceptible individuals. We assume that $\mathrm{u}$ maxis never equal to 1 , since it the model is more realistic from a medical point of view.

The aim is to find the optimal values $u^{*}($.$) of the controls u($.$) such that the associated state$ trajectories $S^{*}, E^{*}, I^{*}, R^{*}$ and $D^{*}$ are solutions of the system in equation $\left(S_{2}\right)$ in the intervention time interval $[0, T$ with initial given conditions:

$$
S(0) \geq 0 \quad E(0) \geq 0 \quad I(0) \geq 0 \quad R(0) \geq 0 \quad D(0) \geq 0
$$

Our goal is to minimize the number of susceptible and infected individuals during the course of an epidemic. Mathematically, for a fixed terminal time $\mathrm{T}$, the problem is to minimize the objective function given by:

$$
J(u(t))=\int_{0}^{T}\left(\alpha S(t)+\rho I(t)+\frac{\varphi}{2} u(t)^{2}\right) \mathrm{dt}
$$

where $\alpha \geq 0, \rho \geq 0, \varphi \geq 0$ denotes weights that balance the size of the terms. In other words, we seek the optimal $u^{*}$ control which considers the fraction of susceptible individuals $(S)$ and the infected individuals $(I)$ and the cost associated with COVID-19 transmission measures. 
The control system in Equation $\left(S_{2}\right)$ of ordinary differential equations in $\mathbb{R}^{5}$ is calculated using the set of admissible control functions given by:

$$
\Omega=\left\{u(.) \in L^{\infty}(0, T) \mid 0 \leq u(.) \leq u_{\max }, \forall t \in[0, T]\right\}
$$

We consider the optimal control problem of determining $\left(S^{*}(),. E^{*}(),. I^{*}(),. R^{*}(),. D^{*}().\right)$ associated to an admissible control $u^{*}($.$) Won the time interval [0, T]$, satisfying Equation $\left(S_{2}\right)$ and the initial conditions of Equation and minimizing the cost functional of Equation (3.1):

$$
J\left(u^{*}(t)\right)=\max _{\Omega} J(u(.))
$$

Note that we are considering a $L^{2}$-cost function: the integrand of the cost functional $J$ is concave with respect to the control $u$. Moreover, the control system of Equation $\left(S_{2}\right)$ is Lipschitz with respect to the state variables $(S, E, I, R, D)$. These properties ensure the existence of an optimal control $u^{*}($.$) of the optimal control problem in Equations \left(S_{2}\right)(3.3)$ (see [6]).

To solve optimal control problems, two approaches are possible: direct and indirect. Direct methods consist of the discretization of the optimal control problem. Indirect methods are based on Pontryagins Maximum Principle to characterize the optimal controls and the optimality system is solved by an iterative method. Using, MATLAB we produce numerical simulations for validation of results of control variables to the solution of a boundary value problem through Pontryagins Maximum.

3.1. Pontryagins Maximum Principle. According to Pontryagins Maximum Principle (see [7]), if $u^{*}($.$) is optimal for Equations \left(S_{2}\right)(3.3)$ with fixed final time $T$, then there exists a nontrivial continuous mapping.

$$
\begin{aligned}
\Phi:[0, T] & \longrightarrow \mathbb{R}^{5} \\
t & \longrightarrow \Phi(t)=\left(\lambda_{1}(t), \lambda_{2}(t), \lambda_{3}(t), \lambda_{4}(t), \lambda_{5}(t)\right)
\end{aligned}
$$

called the adjoint vector, such that

and

$$
S^{\prime}=\frac{\partial H}{\partial \lambda_{1}}, \quad E^{\prime}=\frac{\partial H}{\partial \lambda_{2}}, \quad I^{\prime}=\frac{\partial H}{\partial \lambda_{3}}, \quad R^{\prime}=\frac{\partial H}{\partial \lambda_{4}}, \quad D^{\prime}=\frac{\partial H}{\partial \lambda_{5}}
$$

$$
\lambda_{1}^{\prime}=-\frac{\partial H}{\partial S}, \quad \lambda_{2}^{\prime}=-\frac{\partial H}{\partial E}, \quad \lambda_{3}^{\prime}=-\frac{\partial H}{\partial I}, \quad \lambda_{4}^{\prime}=-\frac{\partial H}{\partial R}, \quad \lambda_{5}^{\prime}=-\frac{\partial H}{\partial D}
$$

where the state equation is

$$
\left\{\begin{array}{l}
f_{1}(S, E, I, R, D)=-\beta(1-u(t)) S(t) I(t) \\
f_{2}(S, E, I, R, D)=\beta(1-u(t)) S(t) I(t)-\eta E(t) \\
f_{3}(S, E, I, R, D)=\eta E(t)-\delta I(t) \\
f_{4}(S, E, I, R, D)=\delta(1-\gamma) I(t) \\
f_{5}(S, E, I, R, D)=\delta \gamma I(t)
\end{array}\right.
$$

The Pontryagin's Maximum Principle provides necessary conditions for an optimal control problem. This principle converts into a problem of minimizing the Hamiltonian, 


$$
H(t, S, E, I, R, D, u, \lambda)=\alpha S(t)+\rho I(t)+\frac{\varphi}{2} u(t)^{2}+\sum_{i=1}^{5} \lambda_{i} f_{i}(S, E, I, R, D)
$$

where

$$
\begin{aligned}
H(t, S, E, I, R, D, u, \lambda)= & \alpha S(t)+\rho I(t)+\frac{\varphi}{2} u(t)^{2} \\
& +\lambda_{1}(-\beta(1-u(t)) S(t) I(t)) \\
& +\lambda_{2}(\beta(1-u(t)) S(t) I(t)-\eta E(t)) \\
& +\lambda_{3}(\eta E(t)-\delta I(t)) \\
& +\lambda_{4}(\delta(1-\gamma) I(t)) \\
& +\lambda_{5}(\delta \gamma I(t))
\end{aligned}
$$

By applying the Pontryagin maximum principle to the optimal control problem in Equations $\left(S_{2}\right)$ 3.3. The following theorem is obtained:

Theorem 3.1. Given optimal control $u^{*} \in \Omega$ and solutions $S^{*}, E^{*}, I^{*}, R^{*}, D^{*}$ of the corresponding state system $\left(S_{2}\right)$, there exists adjoins unctions $\lambda_{1}, \lambda_{2}, \lambda_{3}, \lambda_{4}$ and $\lambda_{5}$ satisfying the following equations:

$$
\left\{\begin{array}{l}
-\lambda_{1}^{\prime}=\alpha+\lambda_{1}\left(-\beta\left(1-u^{*}(t)\right) I^{*}(t)\right)+\lambda_{2}\left(\beta\left(1-u^{*}(t)\right) I^{*}(t)\right) \\
-\lambda_{2}^{\prime}=\eta\left(\lambda_{3}-\lambda_{2}\right) \\
-\lambda_{3}^{\prime}=\rho+\beta\left(1-u^{*}(t)\right) S^{*}(t)\left(\lambda_{2}-\lambda_{1}\right)-\lambda_{3} \delta+\lambda_{4}(\delta(1-\gamma))+\lambda_{5} \delta \gamma \\
-\lambda_{4}^{\prime}=0 \\
-\lambda_{5}^{\prime}=0
\end{array}\right.
$$

with transversally conditions

$$
\lambda_{i}(T)=0, \quad \forall i=1, \cdots, 5
$$

Moreover, the optimal control $u^{*}$ is given by:

$$
u^{*}(t)=\min \left(u_{\max }, \max \left(0,\left(\lambda_{2}-\lambda_{1}\right) \frac{\beta S^{*}(t) I^{*}(t)}{\varphi}\right)\right)
$$

The optimal control $u$ can be solved from the optimality condition,

$$
\frac{\partial H}{\partial u}=\frac{\varphi}{2} u^{*}(t)^{2}+\lambda_{1}(t) \beta S^{*}(t) I^{*}(t)-\lambda_{2}(t) \beta S^{*}(t) I^{*}(t)=0 .
$$

3.2. Numerical Simulation. The numerical algorithm presented below is a semi-implicit finite difference method. We discretize the interval $[0, T]$ at the points $t_{0}=0+i h, i: 0, \ldots, n$, where $h$ is the time step such that $t_{n}=T$.

In our study of the control of the COVID-19, we use the parameters defined in Section 2. Concerning the numerical simulations of the optimal control problem, we have used Matlab/Smulink. We consider $u$ max $=0.5$, representing a misuse of the preventive COVID-19 measures $u($.$) which is the set of admissible controls is given by:$ 
In our study of the control of the COVID-19, we use the parameters defined in Section 2. Concerning the numerical simulations of the optimal control problem, we have used Matlab/Smulink. We consider $u_{\max }=0.5$, representing a misuse of the preventive COVID-19 measures $u($.$) , which is the set of admissible controls is given by$

$$
\Omega=\left\{u(.) \in L^{\infty}(0, T) \mid 0 \leq u(.) \leq 0.5, \forall t \in[0, T]\right\}
$$

with $\mathrm{T}=2$ (years). Figure 4 shows the numerical solution to the optimal control problem of Equations $\left(S_{2}\right)(3.3)$ with the initial conditions and the admissible control set in (3.4).

Figure 5 illustrates the evolution of de Susceptible numbers and Recovered both in without control and in the presence of the control.

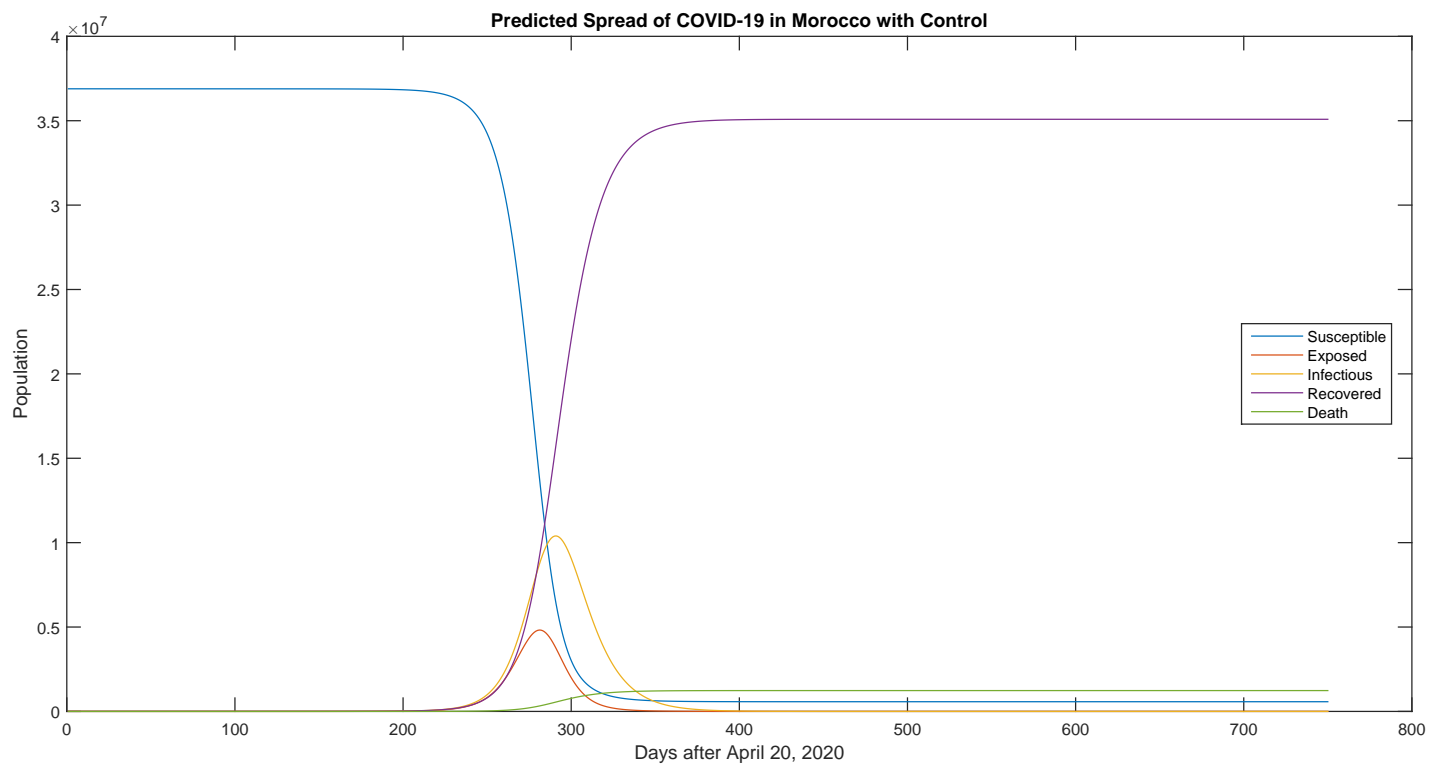

Figure 4. With control - Predicted Spread of COVID-19 in Morocco for 2 years 

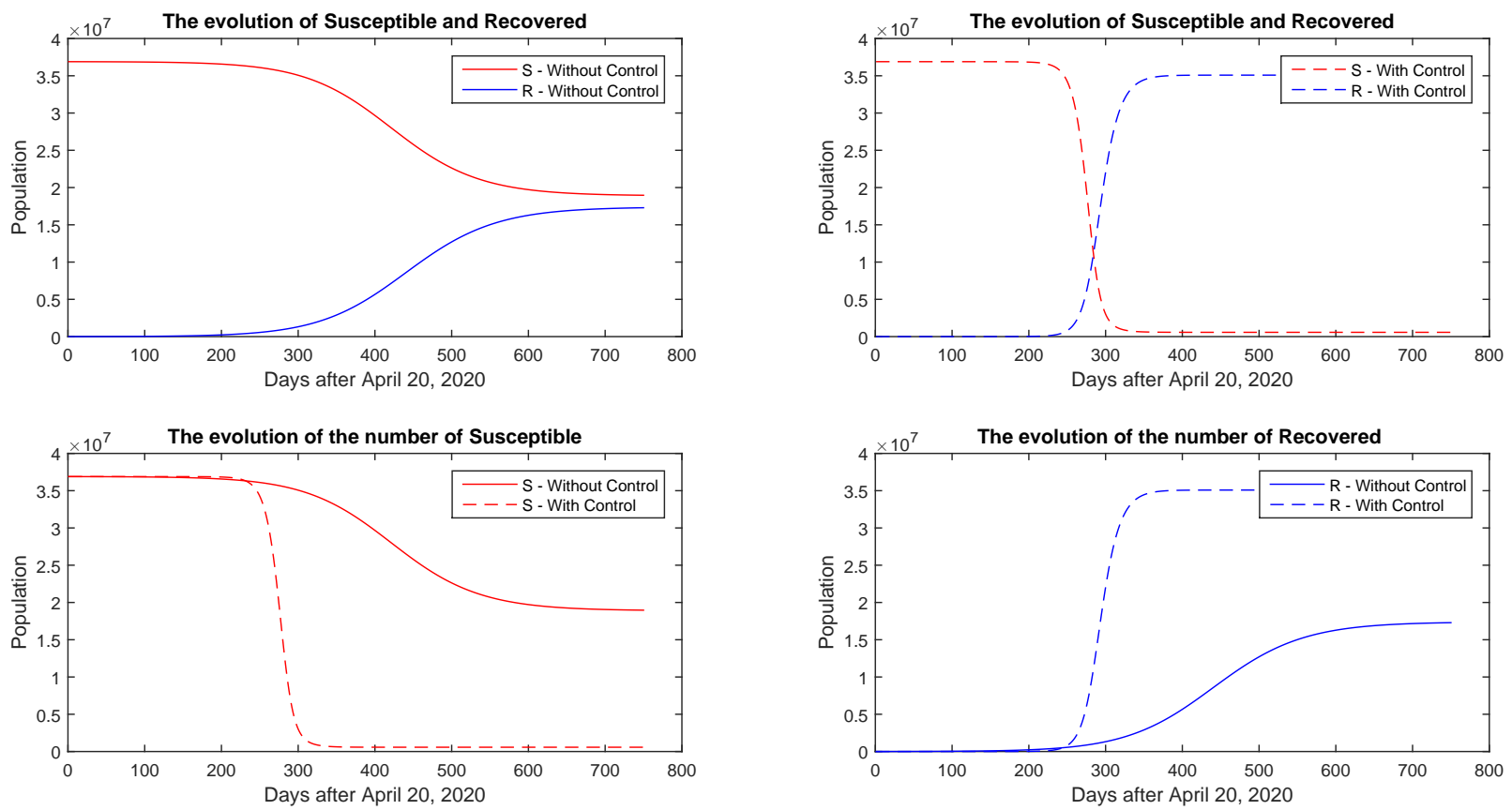

Figure 5

\section{Conclusion}

The COVID-19 infection was first brought into by to Morocco from European countries. The Moroccan governgent has employed a series of rigorous control measures to limited personto-person transmission fof COVID-19 and imposed containment measures to halt the spread of virus among the population. In this article, an effective strategy is implemented to decrease the progression of COVID-19 in Morocco. The findings based on this strategy show that protective measures have played a significant role in limiting the extent of the spread of the disease. Hence, the optimal Control Theory that has been applied based on SEIRDs model suggests applying COVID-19 preventive measures such as physical distancing and wearing masks. The optimal Control Theory is established using Pontryagins Maximum Principle. An expression for the basic reproduction number is derived in terms of control variables. The numerical simulation of the model clearly demonstrates the effectiveness of the strategies in reducing the number of infected individuals in the population in good agreement with our results.

\section{Data Availability}

The disciplinary data used to support the findings of this study have been deposited in the Network Repository (http://networkrepository.com/proOle.php). 


\section{Conflict of Interests}

The authors declare that there is no conflict of interests regarding the publication of this paper.

\section{References}

[1] O. Ifguis, M. El Ghozlani ,F Ammou,A. Moutcine and Z. Abdellah. "Simulation of the Final Size of the Evolution Curve of Coronavirus Epidemic in Morocco using the SIR Model" Hindawi. Journal of Environmental and Public Health. Volume 2020, Article ID 9769267, 5 pages

[2] S. Bentout, A. Chekroun and T. Kuniya. "Parameter estimation and prediction for coronavirus disease outbreak 2019" AIMS Public Health, 7(2): 306318. OI:10.3934/publichealth.2020026 (COVID-19) in Algeria

[3] World Health Organization. Report of the WHO-China Joint Mission on Coronavirus Disease 2019 (COVID-19) Available at: https://www.who.int/docs/default-source/coronavir use/who-china-joint-mission-on-covid19-final-report.pdf (Accessed 13 March 2020).

[4] https://www.worldometers.info/coronavirus/country/morocco/

[5] R. Ait Addi, A. Benksim, M. Amine, M. Cherkaoui "COVID-19 Outbreak and Perspective in Morocco" Electronic Journal of General Medicine 2020, 17(4), em204 e-ISSN: 2516-3507

[6] L. Cesari, OptimizationTheory and Applications; Springer-Verlag: New York, NY, USA, 1983. doi:10.1007/9781-4613-8165-5.

[7] L.S. Pontryagin, V.G. Boltyanskii, R.V. Gamkrelidze, E.F Mishchenko. The Mathematical Theory of Optimal Processes; Translated from the Russian by K. N. Trirogoff; Neustadt, L.W., Ed.; John Wiley and Sons, Inc.: New York, NY, USA, 1962

[8] J. Karrakchou, M. Rachik, and S. Gourari, Optimal control and Infectiology: Application to an HIV/AIDS Model, Applied Mathematics and Computation, (2006), 177:807818.

[9] A. Kouidere, O. Balatif, H. Ferjouchia, A. Boutayeb, and M. Rachik1. Optimal Control Strategy for a Discrete Time to the Dynamics of a Population of Diabetics with Highlighting the Impact of Living Environment. Discrete Dynamics in Nature and Society, Volume 2019, Article ID 6342169, 8 pages.

[10] A. B. Gumel, P. N. Shivakumar, and B. M. Sahai, A mathematical model for the dynamics of HIV-1 during the typical course of infection, Third world congress of nonlinear analysts, (2001), 47:20732083.

[11] World Health Organization. WHO coronavirus disease (covid-2019) situation reports, 2020. https://www.who.int/emergencies/diseases/novel-coronavirus-2019/situation-reports.

[12] S. Zhang, M. Y. Diao, W. Yu, L. Pei, Z. Lin, and D. Chen. Estimation of the reproductive number of novel coronavirus (covid-19) and the probable outbreak size on the diamond princess cruise ship: A data-driven analysis. International Journal of Infectious Diseases, 93:201204, 2020.

[13] T.-M. Chen, J. Rui, Q.-P. Wang, Z.-Y. Zhao, J.-A. Cui, and L. Yin. A mathematical model for simulating the phase-based transmissibility of a novel coronavirus. Infectious Diseases of Poverty, 9(1):18, 2020

[14] W. H. Fleming and R. W. Rishel. Deterministic and Stochastic Optimal Control. Stochastic Modelling and Applied Probability. Springer New York, 2012.

[15] W. Jia, K. Han, Y. Song, W. Cao, S. Wang, S. Yang, J. Wang, F. Kou, P. Tai, J. Li, et al. Extended sir prediction of the epidemics trend of covid-19 in italy and compared with hunan, china. medRxiv, 2020.

[16] Q. Lin, S. Zhao, D. Gao, Y. Lou, S. Yang, S. S. Musa, M. H. Wang, Y. Cai, W. Wang, L. Yang, et al. A conceptual model for the coronavirus disease 2019 (covid-19) outbreak in wuhan, china with individual reaction and governmental action. International journal of infectious diseases, 2020

[17] Elsevier. Novel coronavirus information center, 2020

[18] Springer Nature. Sars-cov-2 and covid-19: A new virus and associated respiratory disease, 2020.

[19] Fanadi M et al., Strategies to control COVID-19 and future pandemics in Africa and around the globe. European Heart Journal 2020,

[20] Liu Z, Magal P, Seydi O, and Webb G, Understanding unreported cases in the 2019-nCov epidemic outbreak in Wuhan, China, and the importance of major public health interventions, MPDI Biology 2020, 9(3), 50. 
[21] The Ministry of Health of Morocco, Communique N10: Morocco announces 1st COVID-19 case, www.covidmaroc.ma 2020.

[22] The Ministry of Health of Morocco, Communique N11: Morocco registers 2nd COVID-19 case, www.covidmaroc.ma 2020.

[23] WHO, (16-24 February 2020) Report of the WHO-China joint mission on Coronavirus disease (COVID-19) 2019.

[24] S WJ, J D. Modeling post-death transmission of Ebola: challenges for inference and opportunities for control. Scientific reports. 2015;(5: 8751). doi:10.1038/srep08751

[25] Chowell G, Hengartner N, Castillo-Chavez C, Fenimore P, Hyman J. The basic reproductive number of Ebola and the effects of public health measures: the cases of Congo and Uganda. Journal of Theoretical Biology. 2004;(229):119126. 